Journal of Patient-Centered

\title{
Innovations in U.S. Health Care Delivery to Reduce Disparities in Maternal Mortality Among African American and American Indian/Alaskan Native Women
}

\author{
Swapna Reddy \\ Nina Patel \\ Mary Saxon \\ Nina Amin \\ Rizwana Biviji
}

Follow this and additional works at: https://aah.org/jpcrr

Part of the Community Health Commons, Community Health and Preventive Medicine Commons, Family Medicine Commons, Maternal and Child Health Commons, Nursing Midwifery Commons, Obstetrics and Gynecology Commons, and the Women's Health Commons

\section{Recommended Citation}

Reddy S, Patel N, Saxon M, Amin N, Biviji R. Innovations in U.S. health care delivery to reduce disparities in maternal mortality among African American and American Indian/Alaskan Native women. J Patient Cent Res Rev. 2021;8:140-5. doi: 10.17294/2330-0698.1793

Published quarterly by Midwest-based health system Advocate Aurora Health and indexed in PubMed Central, the Journal of Patient-Centered Research and Reviews (JPCRR) is an open access, peer-reviewed medical journal focused on disseminating scholarly works devoted to improving patient-centered care practices, health outcomes, and the patient experience. 


\title{
Innovations in U.S. Health Care Delivery to Reduce Disparities in Maternal Mortality Among African American and American Indian/Alaskan Native Women
}

\author{
Swapna Reddy, DrPH, JD, ${ }^{1}$ Nina Patel, MS,${ }^{1}$ Mary Saxon, MS,${ }^{1}$ Nina Amin, ${ }^{2}$ Rizwana Biviji, $\mathrm{PhD}^{1}$ \\ ${ }^{1}$ College of Health Solutions, Arizona State University, Phoenix, AZ; ${ }^{2}$ Phoenix Country Day School, Paradise Valley, AZ
}

\begin{abstract}
Despite spending more on health care than any other country, the United States has the worst maternal mortality rate among all developed nations. African American and American Indian/Alaskan Native women have the worst outcomes by race, representing a stark health disparity within the country. Contributing factors disproportionately experienced by these minority populations include challenges of access to consistent and high-quality prenatal care, prevalence of underlying conditions, toxic stress due to systemic racism, and unconscious bias in health care. While many of these factors lie upstream in the lives of women, and seemingly beyond the scope of the clinical walls, the downstream health care delivery system can serve as a vital part of the solution via innovative practices, community-based collaborations, and by serving as advocates for the communities served. Such alignments between clinicians, community leaders, policymakers, and patients that extend beyond the health system can serve as the missing piece needed within the clinic to reverse the trajectory of maternal mortality for American women, especially those from traditionally underserved populations. ( $J$ Patient Cent Res Rev. 2021;8:140-145.)

Keywords health disparities; maternal mortality; African American; American Indian; provider-patient relationship; health equity; patient focus
\end{abstract}

Maternal mortality is due to a confluence of factors both within and outside of the clinical walls, namely social determinants of health, which result in disparate health outcomes for African American (AA) and American Indian/Alaskan Native (AI/AN) women and their families. These factors include disproportionate rates of preexisting health conditions; limited access to health care, including high-quality prenatal care; and the role of historic, current, and pervasive racism. Far more pernicious social realities point to factors like the role of unconscious biases by providers, lack of trust in health systems by patients of color, and toxic stress due to persistent and systemic racism. ${ }^{1}$

COVID-19 has further pulled back the curtain to lay bare the staggering disparities that we continue to face regarding health outcomes and justice for communities of color in the United States, especially for AA and AI/AN groups. ${ }^{2}$ Globally, maternal morbidity and mortality are considered important indicators of a country's overall

Corresponding author: Swapna Reddy, DrPH, JD, College of Health Solutions, Arizona State University, Phoenix, AZ 85004 (swapna.reddy@asu.edu) health care quality. Despite high health care spending, the United States has some of the worst maternal outcomes in the industrialized world., ${ }^{3,4}$ Its overall maternal mortality rate (MMR) is the highest among all developed nations at 17.4 per 100,000 live births, while AA women have a MMR of 42.4 and AI/AN women a MMR of $30 .{ }^{2.5,6}$ An estimated $60 \%$ of U.S. maternal deaths are preventable, revealing missed opportunities for intervention, especially for $\mathrm{AA}$ and $\mathrm{AI} / \mathrm{AN}$ women. ${ }^{7}$

Since issues within health care have historically been part of the problem, the authors contend that the health care delivery system must also be part of the solution. In this topic synopsis, the contributing factors of access to health care and racism that lead to disproportionate rates of maternal mortality among $\mathrm{AA}$ and $\mathrm{AI} / \mathrm{AN}$ women are briefly reviewed, as are several health care delivery recommendations for reducing these disparities.

\section{Underlying Health Conditions}

The overall health of a woman prior to pregnancy is critical for both maternal and fetal health outcomes. In addition to experiencing disproportionate rates of maternal mortality, $\mathrm{AA}$ and $\mathrm{AI} / \mathrm{AN}$ populations experience higher rates of underlying health conditions, such as heart disease, obesity, and diabetes, that may lead to poor birth outcomes. In fact, of AA women above the age of 20 , 
$48 \%$ have cardiovascular disease (with hypertension by far the most common). ${ }^{8}$ Nearly two-thirds of AA women are considered obese according to their body mass index. ${ }^{9}$

AI/AN populations have lower-rated health status compared to their non-AI/AN counterparts, with a life expectancy that is 5.5 years less than the overall U.S. population. ${ }^{10}$ Compared to White women, AI/AN women are 1.4 times more likely to be diagnosed with gestational hypertension and preeclampsia and 1.5 to 2 times more likely to be diagnosed with gestational diabetes. ${ }^{11}$

\section{Access to Quality Prenatal Care}

Women who receive no prenatal care are 3 to 4 times more likely to die of pregnancy-related complications as compared to women who receive adequate prenatal care. ${ }^{12}$ In $2018,6 \%$ of women reported receiving prenatal care only in the third trimester or receiving no prenatal care. ${ }^{13}$ This number increases to $10 \%$ for $\mathrm{AA}$ and $12 \%$ for $\mathrm{AI} / \mathrm{AN}$ women. ${ }^{14}$ Early initiation of prenatal care and adequate monitoring by providers are important to mitigate severe pregnancy-related complications. ${ }^{5}$ AA women are less likely to be insured, experience greater financial barriers, and have less access to prenatal care. ${ }^{15}$

Disparities in accessing early and continuous prenatal care also exist among women residing in rural underserved areas, which is particularly harmful to AI/AN women, as $40 \%$ live on reservation, rural, or frontier communities that are long distances from care. As such, fewer AI/AN women living in rural communities access prenatal care within the first trimester, compared to White women. ${ }^{16}$ While many AI/AN women are entitled to health care via the Indian Health Services, many challenges persist in accessing care due to barriers like transportation and difficulties taking time off work to access these services. ${ }^{16}$

\section{The Pervasive Role of Racism}

Racial/ethnic disparities in maternal mortality can be attributed to factors such as racism that exist at multiple levels. ${ }^{17}$ This is amplified by high rates of poverty, housing challenges, job discrimination, and social isloation. ${ }^{17,18}$ It is important to note that it is not race itself, but the underlying racism that $\mathrm{AA}$ and $\mathrm{AI} / \mathrm{AN}$ women experience on a systemic basis, that contributes to poor pregnancy outcomes and higher MMR. ${ }^{19-22}$ The accumulation of chronic stress over a woman's life course, due to racism, can result in poor perinatal health outcomes like preterm births and low-birth-weight infants. ${ }^{22,23}$ This is especially true among AA women who disproportionately experience the compounding effects of racism that result in an increased allostatic load (ie, the cumulative effect of chronic stress). ${ }^{17,24}$ While educational attainment (an indicator of structural racism) is generally associated with severe maternal morbidity, ${ }^{19}$ racial/ethnic disparities in maternal outcomes persist regardless of a woman's socioeconomic or educational status, ${ }^{17}$ as collegeeducated AA mothers are still more likely to suffer severe complications in pregnancy or childbirth than White women who never graduated from high school, reflecting gaps related to racism and bias. ${ }^{25}$

$\mathrm{AI} / \mathrm{AN}$ communities also have experienced centuries of racism and discrimination in the form of genocide, forced migration, and cultural erasure. ${ }^{18,21}$ As a result, $\mathrm{AI} / \mathrm{AN}$ women are twice as likely as their White counterparts to experience emotional or physiological distress. ${ }^{18} \mathrm{AI} / \mathrm{AN}$ women are at a higher risk of experiencing sexual and interpersonal violence during their lifetime; which has been linked to preterm births, delayed or no prenatal care, and unintentional pregnancy. ${ }^{11,21}$ The history of forced sterilization and infant separation policies have additionally resulted in distrust between $\mathrm{AI} / \mathrm{AN}$ women and their care providers, thereby negatively impacting patient-provider relationships. ${ }^{11}$

Unconscious biases in the form of attitudes and stereotypes held by health care providers may negatively impact their clinical judgment while caring for minority patients. ${ }^{22,26,27}$ Examples include the routine dismissal of the intensity of symptoms expressed by AA women by health professionals, leading to delays in treatment or poorer outcomes, ${ }^{28}$ and studies finding White medical students incorrectly rating AA patients' pain lower than that of White patients, leading to less accurate treatment recommendations. ${ }^{29}$ Similarly, more than one-fourth of $\mathrm{AI} / \mathrm{AN}$ women have faced discrimination in accessing health care services. ${ }^{30}$

Taking into account these prevalent contributing factors - specifically health care access and the role of racism in health care - a paradigm shift is needed to reimagine the health care delivery team as part of the solution.

\section{Health Care Delivery Models - Addressing Unconscious Biases}

To effectively address these contributing factors, systems must be designed with standardized safety protocols to mitigate the impact of human errors and unconscious biases on the delivery of care to AI/AN and AA women. Initiatives working to standardize health care delivery will likely improve care across all types of health venues but in particular lower-performing hospitals that serve disproportionate rates of minority women. ${ }^{31}$ The implementation of safety bundles - for example, obstetric and postpartum hemorrhage carts - are critical steps toward improving care to women within a clinical setting. ${ }^{31}$ Additionally, protocols, checklists, 
triggers (eg, maternal early warning criteria), simulation training, coordinated care practices, clinician training, credentialing, and the promotion of a safety culture have all been recommended in recent reviews to reduce maternal complications and deaths. ${ }^{32}$ The following paragraphs highlight some current examples of state and national efforts.

A successful approach to address provider bias has been shown in California - one of the states leading the charge to reduce the increasing national trend - through the California Maternal Quality Care Collaborative (CMQCC). This 2006 initiative established a maternal mortality review committee that uncovered that maternal survival rates could be increased by addressing hemorrhage and preeclampsia. ${ }^{33}$ Evidence shows that the majority of maternal deaths from these complications can be prevented through early detection and preparedness with a series of well-researched protocols and checklists to eliminate providers' unconscious bias; see for instance the "hemorrhage cart," which is filled with checklists, intravenous lines, and other equipment needed by the health care team in an emergency. ${ }^{34}$ With these health care delivery solutions, California has managed to cut its rate of maternal mortality by more than half within 12 years, with AA maternal mortality on the decline. ${ }^{35}$

Another example of a health care delivery-based solution can be seen in North Carolina, in which a primary health care model called Pregnancy Medical Home allows women to have their medical, behavioral, and social health needs met within a single location. ${ }^{36}$ In this group prenatal care model, women are provided with both medical care and expanded social and community support. There is evidence to support that maternal medical home models have been improving outcomes for pregnant women through risk screening, communitybased care management, and care pathways - but also by addressing nonmedical issues that can put mother and child at risk, such as addiction, domestic abuse, and lack of secure housing and healthy food. ${ }^{38}$

On a national level, the Council on Patient Safety in Women's Health Care and the Alliance for Innovation in Maternal Health recently published the "Reduction of Peripartum Racial/Ethnic Disparities" patient safety bundle, which provides actionable steps that state-level institutions and clinicians can implement to reduce disparities in maternal mortality and health disparities. The bundle considers steps across the care continuum, including clinician education regarding unconscious biases, systems-level quality improvement techniques, and best practices for building a culture of equity. ${ }^{39}$

\section{Community-Based Solutions - Providing Cultural Sensitivity and Access to Care}

Community solutions offer promising results in bridging access to care by bringing culturally sensitive approaches and trusted caregivers into health care. Cultural sensitivity in health care includes awareness about the cultural customs and norms an individual may possess regarding their health and treatment. ${ }^{40}$ By including community caregivers like doulas and midwives on the health care team, care becomes higher quality and the needs of the mother are more likely to be met because these caregivers provide cultural sensitivity along with earlier, continuous, and affordable care. ${ }^{37}$ While the doula and midwife serve different purposes in the community and have different educational backgrounds, both act as vital tools to either provide care beyond the hospital setting or a culturally sensitive approach to care within clinic walls.

Medical School Training and Education. Other initiatives have focused on addressing conscious and unconscious bias at the medical school level. The University of Texas Dell Medical School (Austin, TX) is notable for its requirement that all second-year medical students must participate in training on unconscious biases, beginning with a conversation about conscious and unconscious bias and ending with an online test to help students pinpoint their own implicit biases and facilitate class discussion on strategies to address them in practice. ${ }^{26}$ Additionally, at Mayo Clinic Alix School of Medicine (Rochester, MN), medical students participate in an unconscious bias session in the first 2 weeks of the first year. ${ }^{41}$ This content is part of a required 4-year science of health care delivery curriculum that includes education on cultural humility, health care disparities, local community resources to help reduce disparities and increase health equity, racism and mistrust in health care, cross-cultural communication, and equitable care. ${ }^{41}$ While training in of itself does not necessarily eliminate bias, research has shown that it is an important step toward better understanding, communication, and relationshipbuilding between patients and providers. ${ }^{32}$

Cultural Sensitivity. The practice of AI/AN doulas is a beneficial approach to address racism in health care by integrating cultural practices in birthing experiences and improving $\mathrm{AI} / \mathrm{AN}$ maternal child health outcomes. ${ }^{42} \mathrm{~A}$ 2007 study identified that $49.6 \%$ of AI/AN women wanted their cultural practices integrated into their birthing experience, and $44.1 \%$ felt their prenatal provider was not culturally sensitive. ${ }^{42} \mathrm{AI} / \mathrm{AN}$ doula practice includes cultural components, such as ceremonial singing and prayer, which makes their care a likely source of cultural sensitivity for AI/AN women during their prenatal and birthing experience. ${ }^{43}$ The inclusion of cultural practices 
by doulas from similar communities helps to bridge larger concerns related to trust in the health system for AI/AN women. ${ }^{43}$ Additionally, AA midwives are important in mitigating racial bias in the health system by advocating for AA mothers in prenatal, delivery, and postnatal care. ${ }^{44}$ Specifically, the midwife is able to provide full antenatal care, including parenting classes, clinical examinations, screenings, and advocating for one's prediscussed choices in labor and delivery. ${ }^{37}$

Access to Care and Support. There is a well-demonstrated demand for health professionals who understand the needs of individuals of color. ${ }^{44}$ When AA or AI/AN patients are cared for by health professionals of their own race, ethnicity, or cultural background, otherwise known as concordance, patient experience and outcomes tend to improve as patients often perceive that they are better heard/understood and have a shared experience. ${ }^{44,45}$ In the absence of racial and ethnic concordance between health professionals and AA or AI/AN mothers, it is critical for providers to be better informed of the social determinants of health and systemic barriers faced by their patients that can impact maternal mortality outcomes. Midwives and doulas within the health care team can play an important role in bridging the gap by fostering more shared understanding, trust, access, and cultural practices, which can contribute to improved maternal fetal health outcomes and reduced health care costs..$^{37,46}$

\section{Policy Efforts}

Expanding and Enhancing Access to Care. Policy implementation at local, state, and federal levels can serve as key solutions to bridge systemic inequities in maternal health at population levels. Medicaid is the insurer for $45 \%$ of all births nationally, with the program covering $66 \%$ of all births to AA women. Most Medicaidfinanced births take place in hospitals nationally; in 23 states, more than $10 \%$ are overseen by a certified nurse midwife. ${ }^{47,48}$ Strengthening the Medicaid program to expand the program in all states - including more holistic coverage during pregnancy, extending postpartum coverage, enhancing benefits for home visits, midwives, and doulas, and developing value-based payments rewarding positive outcomes - is critical to closing gaps in maternal outcomes and increasing equity in vulnerable communities. ${ }^{49,50}$

Reducing Unconscious Bias. Policies aimed at reducing unconscious bias among providers and health systems also can play an important role in improving outcomes in health care delivery. An example is California Dignity in Pregnancy and Childbirth Act Senate Bill 464, which provides comprehensive clinical training to reduce unconscious bias among providers and focuses on improving data collection during pre- and postnatal care. ${ }^{51}$ This bill works in conjunction with other state initiatives like the CMQCC to build shared understanding and bridge health inequities through clinician training. Similar enacted legislation at the federal level (eg, Social Determinants for Moms Act, Kira Johnson Act, Protecting Moms Who Served Act, Perinatal Workforce Act, Data to Save Moms Act, Moms MATTER Act, Justice for Incarcerated Moms Act, Tech to Save Moms Act, IMPACT to Save Moms Act) can serve as vital tools in reducing the role of racism and bias in maternal health and childbirth within the clinical walls. ${ }^{52}$

\section{Summary}

The integrated efforts of many frontline health care professionals, community leaders, and policymakers showcase promising steps toward bridging the continuum of health disparities and building on shared understanding to improve maternal outcomes in the United States. To move the needle forward on maternal outcomes, especially for the African American and American Indian/Alaskan Native communities disproportionately and continuously experiencing poor health outcomes, all stakeholders need to think, partner, and act beyond the clinical walls to forge a more equitable and effective health care delivery system within those walls.

\section{Patient-Friendly Recap}

- The United States has unacceptably high maternal mortality rates, especially for African American and American Indian mothers.

- Authors reviewed several factors - including prevalence of underlying conditions, racism-induced stress, unconscious bias in health care, and lack of quality prenatal care - that contribute to this health disparity.

- Strategies for improving maternal health in minority populations include fostering health care/community collaborations, embracing the contributions of culturally sensitive midwives or doulas, and expanding proven legislative programs.

\section{Conflicts of Interest}

None.

\section{References}

1. Hostetter M, Klein S; on behalf of The Commonwealth Fund. In focus: reducing racial disparities in health care by confronting racism. Published September 27, 2018; accessed November 25, 2020. https://www.commonwealthfund.org/publications/2018/ sep/focus-reducing-racial-disparities-health-care-confrontingracism 
2. Artiga S, Corallo B, Pham O; on behalf of Kaiser Family Foundation. Racial disparities in COVID-19: key findings from available data and analysis. Published August 17, 2020; accessed November 29, 2020. https://www.kff.org/racialequity-and-health-policy/issue-brief/racial-disparities-covid19-key-findings-available-data-analysis/

3. MacDorman M, Declercq E. The failure of United States maternal mortality reporting and its impact on women's lives. Obstet Gynecol Surv. 2018;73:615-6. CrossRef

4. Gunja MZ, Tikkanen R, Seervai S, Collins SR; on behalf of The Commonwealth Fund. What is the status of women's health and health care in the U.S. compared to ten other countries? Published December 19, 2018; accessed June 10, 2020. https://www.commonwealthfund.org/publications/ issue-briefs $/ 2018 / \mathrm{dec} /$ womens-health-us-compared-tenother-countries

5. Centers for Disease Control and Prevention, National Center for Health Statistics. First data released on maternal mortality in over a decade [press release]. Released January 30, 2020; accessed July 17, 2020. https://www.cdc.gov/nchs/pressroom/ nchs_press_releases/2020/202001_MMR.htm

6. Centers for Disease Control and Prevention, National Center for Health Statistics. Maternal mortality. Page last reviewed November 9, 2020; accessed June 10, 2020. https://www.cdc. gov/nchs/maternal-mortality/index.htm

7. Petersen EE, Davis NL, Goodman D, et al. Vital signs: pregnancy-related deaths, United States, 2011-2015, and strategies for prevention, 13 States, 2013-2017. MMWR Morb Mortal Wkly Rep. 2019;68:423-9. CrossRef

8. Benjamin EJ, Virani SS, Callaway CW, et al. Heart Disease and Stroke Statistics-2018 Update: a report from the American Heart Association. Circulation. 2018;137:e67-492. CrossRef

9. Agyemang P, Powell-Wiley TM. Obesity and black women: special considerations related to genesis and therapeutic approaches. Curr Cardiovasc Risk Rep. 2013;7:378-86. CrossRef

10. Indian Health Service. Fact sheets: disparities. Published October 2019; accessed November 23, 2020. https://www.ihs. gov/newsroom/factsheets/disparities/

11. National Partnership for Women \& Families. American Indian and Alaska Native women's maternal health: addressing the crisis. Published October 2019; accessed October 23, 2020. https://www.nationalpartnership.org/our-work/resources/ health-care/maternity/american-indian-and-alaska.pdf

12. MacDorman MF, Declercq E. Trends and state variations in out-of-hospital births in the United States, 2004-2017. Birth. 2019;46:279-88. CrossRef

13. The Annie E. Casey Foundation KIDS COUNT Data Center. Births to women receiving late or no prenatal care in the United States. Updated February 2021; accessed June 10, 2020. https://datacenter.kidscount.org/data/tables/11-births-towomen-receiving-late-or-no-prenatal-care

14. Child Trends. Late or no prenatal care. Published 2019; accessed June 10, 2020. https://www.childtrends.org/indicators/late-orno-prenatal-care

15. National Partnership for Women \& Families. Black women's maternal health: a multifaceted approach to addressing persistent and dire health disparities. Published April 2018; accessed November 23, 2020. https://www.nationalpartnership. org/our-work/resources/health-care/maternity/black-womensmaternal-health-issue-brief.pdf
16. Hanson JD. Understanding prenatal health care for American Indian women in a Northern Plains tribe. J Transcult Nurs. 2012;23:29-37. CrossRef

17. Barr DA. Health Disparities in the United States: Social Class, Race, Ethnicity, and the Social Determinants of Health, Third Edition. Johns Hopkins University Press; 2019.

18. Truschel L, Novoa C; on behalf of Center for American Progress. American Indian and Alaska Native maternal and infant mortality: challenges and opportunities. Published July 9, 2018; accessed June 18, 2020. https://www.americanprogress. org/issues/early-childhood/news/2018/07/09/451344/ american-indian-alaska-native-maternal-infant-mortalitychallenges-opportunities/

19. Yan Liu S, Fiorentini C, Bailey Z, Huynh M, McVeigh K, Kaplan D. Structural racism and severe maternal morbidity in New York State. Clin Med Insights Womens Health. 2019;12(Jan-Dec 2019). CrossRef

20. Mendez DD, Hogan VK, Culhane JF. Institutional racism, neighborhood factors, stress, and preterm birth. Ethn Health. 2014;19:479-99. CrossRef

21. Raglan GB, Lannon SM, Jones KM, Schulkin J. Racial and ethnic disparities in preterm birth among American Indian and Alaska Native women. Matern Child Health J. 2016;20:16-24. CrossRef

22. Villavicencio JC, McHugh KW, Edmonds BT. Overview of US maternal mortality policy. Clin Ther. 2020;42:408-18. CrossRef

23. Maternal Health Task Force. Maternal health in the United States. Accessed July 20, 2020. https://www.mhtf.org/topics/ maternal-health-in-the-united-states/

24. Wazlowski S; on behalf of American Public Health Association's Public Health Newswire. APHA kicks off webinar series with "Racism: The Ultimate Underlying Condition." Published June 12, 2020; accessed June 15, 2020. http://www. publichealthnewswire.org/?p=racial-equity-webinar-1

25. New York City Department of Health and Mental Hygiene. Severe maternal morbidity in New York City, 2008-2012. Published 2016; accessed July 16, 2020. https://www1.nyc. gov/assets/doh/downloads/pdf/data/maternal-morbidityreport-08-12.pdf

26. Jaynes $C$; on behalf of Health Affairs. To save new mothers, we need to rethink care delivery. Published October 31, 2019; accessed November 23, 2020. https://www.healthaffairs.org/ do/10.1377/hblog20191024.413116/full/

27. Staats C, Capatosto K, Wright RA, Contractor D. State of the science: implicit bias review 2015. Accessed July 10, 2020. http://kirwaninstitute.osu.edu/wp-content/ uploads/2015/05/2015-kirwan-implicit-bias.pdf

28. Roeder A. America is failing its black mothers. Harvard Public Health. Published Winter 2019; accessed November 23, 2020. https://www.hsph.harvard.edu/magazine/magazine_article/ america-is-failing-its-black-mothers/

29. Hoffman KM, Trawalter S, Axt JR, Oliver MN. Racial bias in pain assessment and treatment recommendations, and false beliefs about biological differences between blacks and whites. Proc Natl Acad Sci U S A. 2016;113:4296-301. CrossRef

30. Harvard T.H. Chan School of Public Health. Poll finds more than one-third of Native Americans report slurs, violence, harassment, and being discriminated against in the workplace. Published November 14, 2017; accessed July 16, 2020. https:// www.hsph.harvard.edu/news/press-releases/poll-nativeamericans-discrimination/ 
31. Shah Arora K, Shields LE, Grobman WA, D’Alton ME, Lappen JR, Mercer BM. Triggers, bundles, protocols, and checklists what every maternal care provider needs to know. Am J Obstet Gynecol. 2016;214:444-51. CrossRef

32. Howell E. Reducing disparities in severe maternal morbidity and mortality. Clin Obstet Gynecol. 2018;61:387-99. CrossRef

33. California Maternal Quality Care Collaborative. Who we are. Accessed June 20, 2020. https://www.cmqcc.org/who-we-are

34. Montagne R; on behalf of National Public Radio. To keep women from dying in childbirth, look to California. Published July 29, 2018; accessed July 20, 2020. https://www.npr. org/2018/07/29/632702896/to-keep-women-from-dying-inchildbirth-look-to-california

35. California Maternal Quality Care Collaborative. CA-PAMR (maternal mortality review). Accessed June 20, 2020. https:// www.cmqcc.org/research/ca-pamr-maternal-mortality-review

36. Community Care of North Carolina; Pregnancy Medical Home. Pregnancy medical home: improving maternal \& infant outcomes in the Medicaid population. Accessed December 10, 2020. https://www.communitycarenc.org/what-we-do/clinicalprograms/pregnancy-medical-home

37. McGowan K; on behalf of Maternal Health Task Force's MHTF Blog. Midwives can significantly reduce maternal mortality, but they need support. Published May 5, 2016; accessed June 21, 2020. https://www.mhtf.org/2016/05/05/ midwives-can-significantly-reduce-maternal-mortality-butthey-need-support/

38. Zephyrin L; on behalf of STAT. Pregnancy-related deaths reflect how implicit bias harms women. We need to fix that. Published July 10, 2019; accessed December 10, 2020. https:// www.statnews.com/2019/07/10/pregnancy-related-deathsimplicit-bias/

39. Council on Patient Safety in Women's Health Care. Patient safety bundle. Reduction of peripartum racial/ethnic disparities. Published October 2016; accessed December 10, 2020. https://safehealthcareforeverywoman.org/wp-content/ uploads/Reduction-of-Peripartum-Disparities-Bundle.pdf

40. Georgetown University Health Policy Institute. Cultural competence in health care: Is it important for people with chronic conditions? Published February 13, 2019; accessed December 10, 2020. https://hpi.georgetown.edu/cultural/

41. Reddy S, Starr S, Hayes S, et al. Implicit bias curricula in medical school: student and faculty perspectives. Published January 15, 2020; accessed December 10, 2020. https://www. healthaffairs.org/do/10.1377/hblog20200110.360375/full/

42. Changing Woman Initiative. White Shell Woman Homebirth Services. Accessed June 25, 2020. http://www. changingwomaninitiative.com/white-shell-woman-homebirthservices.html
43. Ireland S, Montgomery-Andersen R, Geraghty S. Indigenous doulas: a literature review exploring their role and practice in western maternity care. Midwifery. 2019;75:52-8. CrossRef

44. Terreri $\mathrm{C}$; on behalf of Lamaze International. Black History Month: the importance of black midwives, then, now and tomorrow. Published February 22, 2019; accessed August 4, 2020. https://www.lamaze.org/Connecting-the-Dots/blackhistory-month-the-importance-of-black-midwives-then-nowand-tomorrow-1

45. Tanne JH. Patients are more satisfied with care from doctors of same race. BMJ. 2002;325(7372):1057.

46. Kozhimannil KB, Hardeman RR, Attanasio LB, BlauerPeterson C, O'Brien M. Doula care, birth outcomes, and costs among Medicaid beneficiaries. Am J Public Health. 2013;103:e113-21. CrossRef

47. Medicaid and CHIP Payment and Access Commission. Medicaid's role in financing maternity care. Published January 2020; accessed November 29, 2020. https://www.macpac.gov/ wp-content/uploads/2020/01/Medicaid $\%$ E2\%80\%99s-Rolein-Financing-Maternity-Care.pdf

48. Markus AR, Andres E, West KD, Garro N, Pellegrini C. Medicaid covered births, 2008 through 2010, in the context of the implementation of health reform. Womens Health Issues. 2013;23:e273-80 CrossRef

49. Khanal P, McGinnis T, Zephyrin L; on behalf of The Commonwealth Fund. Tracking state policies to improve maternal health outcomes. Published November 19, 2020; accessed November 29, 2020. https://www.commonwealthfund. org/blog/2020/tracking-state-policies-improve-maternalhealth-outcomes

50. Waldrop T; on behalf of Center for American Progress. Improving women's health outcomes through payment and delivery system reform. Published June 28, 2019; accessed July 1，2020. https://www.americanprogress.org/issues/ healthcare/reports/2019/06/28/471445/improving-womenshealth-outcomes-payment-delivery-system-reform/

51. California Legislative Information. SB-464 California Dignity in Pregnancy and Childbirth Act. Published October 8, 2019; accessed June 20, 2020. https://leginfo.legislature.ca.gov/ faces/billNavClient.xhtml?bill_id=201920200SB464

52. Underwood L; on behalf of Health Affairs. Saving moms, saving lives. Published April 27, 2020; accessed December 10, 2020. https://www.healthaffairs.org/do/10.1377/ hblog20200423.488851/full/

(C) 2021 Advocate Aurora Health, Inc. 